\title{
ORIGINAL
}

\section{Fibrobroncoscopia en una unidad de vigilancia intensiva respiratoria}

\author{
C.M. Lucena ${ }^{a}$, P. Martínez-Olondris ${ }^{b}$, J.R. Badia ${ }^{a}$, A. Xaubet $^{a}$, M. Ferrer ${ }^{a}$, \\ A. Torres ${ }^{a}$ y C. Agustí ${ }^{a, *}$
}

a Servicio de Neumología, Instituto Clínico del Tórax, Hospital Clínic de Barcelona, Institut d'Investigacions Biomèdiques August Pi i Sunyer (IDIBAPS), Universitat de Barcelona (UB), Ciber de Enfermedades Respiratorias (CIBERES), Barcelona, España

b Servicio de Neumología, Hospital Plató, Barcelona, España

Recibido el 21 de julio de 2011; aceptado el 4 de noviembre de 2011

Disponible en Internet el 22 de diciembre de 2011

\author{
PALABRAS CLAVE \\ Fibrobroncoscopia; \\ Unidad de vigilancia \\ intensiva; \\ Infiltrados \\ pulmonares; \\ Inmunosupresión; \\ Lavado \\ broncoalveolar
}

\begin{abstract}
Resumen
Objetivo: Describir las indicaciones, rentabilidad diagnóstica y complicaciones de la fibrobroncoscopia (FBS) en una unidad de vigilancia intensiva respiratoria (UVIR).

Diseño: Estudio prospectivo observacional.

Ámbito: UVIR de 6 camas en un hospital universitario de tercer nivel.

Pacientes: Pacientes admitidos en una UVIR a los que se les realizó una FBS.

Intervenciones: Ninguna.

Variables de interés: Indicaciones y complicaciones de la FBS, técnicas endoscópicas realizadas y tiempo empleado en la FBS.

Resultados: Se realizaron 107 (23\%) FBS a 69 de los 297 pacientes admitidos en la UVIR. El $68 \%$ de las FBS se practicaron a pacientes con ventilación mecánica. La FBS se realizó con fines diagnósticos en 88 ocasiones (82\%) y terapéuticos en 19 (18\%). La indicación más frecuente para la FBS diagnóstica fue el estudio de infiltrados pulmonares (44 casos; 50\%), particularmente en pacientes inmunodeprimidos (24 casos; $27 \%$ ). Para esta indicación, la rentabilidad diagnóstica de la FBS fue significativamente mejor en los pacientes inmunodeprimidos, respecto a los inmunocompetentes ( $48 \%$ vs $30 \%$; $p<0,01$ ). La FBS no causó complicaciones mayores; únicamente se observó un descenso significativo en la $\mathrm{PaO}_{2} / \mathrm{FiO}_{2}(182 \pm 74$ vs $163 \pm 79 ; \mathrm{p}<0,005)$ cuando se realizó un lavado broncoalveolar. La mortalidad global en la UVIR fue del 14\%; del 25\% en los pacientes que precisaron FBS y del $45 \%$ en aquellos que precisaron FBS adicionales.

Conclusiones: La FBS es un procedimiento seguro y rápido que se utiliza con frecuencia en la UVIR y que contribuye significativamente al manejo clínico. Los pacientes de la UVIR que requieren FBS adicionales tienen una elevada mortalidad.

(c) 2011 Elsevier España, S.L. y SEMICYUC. Todos los derechos reservados.
\end{abstract}

\footnotetext{
* Autor para correspondencia.

Correo electrónico: cagusti@clinic.ub.es (C. Agustí).
} 


\section{KEYWORDS}

Fiberoptic bronchoscopy; Intensive care unit; Pulmonary infiltrates; Immunosuppression; Bronchoalveolar lavage

\section{Fiberoptic bronchoscopy in a respiratory intensive care unit}

\begin{abstract}
Objective: To describe the indications, diagnostic performance and safety of fiberoptic bronchoscopy (FOB) performed in a respiratory intensive care unit (RICU).

Design: A prospective, observational study was carried out.

Setting: A 6-bed RICU in a tertiary university hospital.

Patients: Patients admitted to RICU who required FOB.

Interventions: None.

Main measurements: FOB indications and complications, endoscopic procedures, time required to perform FOB.

Results: Sixty-nine out (23\%) of the 297 patients admitted to the RICU underwent a total of 107 FOB. Sixty-eight percent of FOB were performed in patients on mechanical ventilation. FOB was performed for diagnostic and therapeutic purposes in $88(82 \%)$ and 19 cases (18\%), respectively. The study of pulmonary infiltrates was the main indication for diagnostic FOB (44 cases; $50 \%$ ), particularly in immunocompromised patients (24 cases; $27 \%$ ). In immunocompromised patients the diagnostic performance of FOB was significantly higher than in immunocompetent subjects (48\% vs $30 \%$; $\mathrm{p}<0.01)$. No major complications were recorded. Only a significant drop in $\mathrm{PaO}_{2} / \mathrm{FiO}_{2}$ ratio was observed $(182 \pm 74$ vs $163 \pm 79 ; \mathrm{p}<0.005)$ in patients undergoing bronchoalveolar lavage. Overall mortality in patients in the RICU was $14 \%$. In patients requiring a single FOB procedure, mortality was $25 \%$, versus $45 \%$ among those requiring more than one FOB procedure.

Conclusions: These results show that FOB is used commonly in the RICU. It is a safe and fast procedure that contributes significantly to clinical management. Patients requiring additional FOB during admission to the RICU show high mortality.

(C) 2011 Elsevier España, S.L. and SEMICYUC. All rights reserved.
\end{abstract}

\section{Introducción}

La fibrobroncoscopia (FBS) es una técnica que se utiliza con frecuencia en las unidades de cuidados intensivos (UCI) con propósitos diagnósticos y/o terapéuticos. Hasta la fecha los datos disponibles respecto a las indicaciones, seguridad, rentabilidad diagnóstica e influencia en el curso clínico y pronóstico son escasos y basados en series antiguas ${ }^{1,2}$. En los últimos años, una serie de mejoras en el manejo del paciente crítico, la optimización de las modalidades de ventilación y las técnicas de sedación han permitido ampliar de forma significativa los criterios de admisión en las UCI. Así, es habitual el ingreso de pacientes oncológicos e inmunodeprimidos que hace tan solo unos años no eran admitidos en las $\mathrm{UCl}^{3,4}$ y que frecuentemente presentan complicaciones pulmonares que requieren de una FBS para su diagnóstico.

Aunque la FBS se considera una técnica segura, diversos estudios han demostrado que la introducción del fibrobroncoscopio en el tubo orotraqueal puede alterar parámetros de ventilación y provocar cambios hemodinámicos y en el intercambio de gases ${ }^{5-7}$. A pesar de que generalmente la tolerancia a un lavado broncoalveolar (LBA) es buena, en los pacientes intubados se puede observar con frecuencia un deterioro en la oxigenación arterial ${ }^{8}$. Por otro lado, las indicaciones para la realización de una FBS y la incidencia y el tipo de posibles efectos adversos pueden variar dependiendo del tipo de población estudiada9. En este sentido, la alta prevalencia de pacientes con enfermedad pulmonar admitidos en una unidad de vigilancia intensiva respiratoria (UVIR) debería acarrear a priori mayores riesgos y un mayor índice de complicaciones respiratorias. Hasta el momento no existen publicaciones donde se describa el uso de la FBS en las UVIR.

Con el objetivo de describir las indicaciones, seguridad, rentabilidad diagnóstica e influencia de la FBS en el curso clínico en una UVIR, hemos realizado un estudio prospectivo observacional donde se han incluido todos los procedimientos broncoscópicos llevados a cabo durante un año en una UVIR, dotada de seis camas, en un hospital de tercer nivel.

\section{Métodos}

Estudio prospectivo observacional en el que se incluyeron todos los pacientes a los que se les realizó una FBS en la UVIR entre junio de 2008 y junio de 2009. La indicación de la FBS fue establecida por su médico responsable. Entre las contraindicaciones para llevar a cabo la FBS se incluyeron las alteraciones de la coagulación que no fueron corregidas tras la transfusión de plasma, un recuento plaquetario $<50 \times 10^{6} / \mathrm{uL}$, la hipoxemia severa refractaria a oxigenoterapia $\left(\mathrm{PaO}_{2}<60 \mathrm{mmHg}\right.$ con $\left.\mathrm{FiO}_{2} \geq 0,8\right)$, la inestabilidad hemodinámica a pesar del uso de aminas vasoactivas, la isquemia aguda y las arritmias cardíacas no controladas. El estudio fue realizado siguiendo las guías de FBS diagnóstica ${ }^{10}$.

\section{Procedimiento y monitorización}

Se utilizaron bronscoscopios de 4,9 mm de diámetro externo (FB15-V Pentax Europe GMBH). Todos los pacientes llevaban un catéter venoso y se mantenía monitorización continua 
de la presión sanguínea, del ritmo cardíaco y de la saturación de oxihemoglobina mediante pulsioxímetro, durante el procedimiento y hasta una hora después de la finalización del mismo. Se obtuvieron muestras de sangre arterial que fueron valoradas in situ mediante un analizador de gases en sangre (Radiometer $A B L 30$ ) cinco minutos antes y una hora después del procedimiento. La FBS fue realizada en la UVIR y supervisada por uno de los endoscopistas senior (CA, AX). La exploración endoscópica se practicaba bajo sedación con propofol endovenoso (1-2 mg/Kg) o midazolam $(0,1 \mathrm{mg} / \mathrm{Kg})$ siendo necesario el uso de fentanilo y/o pancuronio en algunas ocasiones. La dosis de medicación variaba individualmente con el objetivo de conseguir una correcta oxigenación y la adaptación del paciente a la ventilación. El broncoscopio se introducía a través de la nasofaringe, la traqueostomía, el tubo orotraqueal (de $8 \mathrm{~mm}$ de diámetro) 0 la mascarilla laríngea. En los últimos tres casos se usaba un adaptador para mantener la ventilación con presión positiva. Los pacientes que precisaban ventilación mecánica se mantenían en modo volumen control. El límite de presión del ventilador se aumentaba para asegurar un volumen corriente adecuado durante cada ciclo respiratorio. $\mathrm{La} \mathrm{FiO}_{2}$ se incrementaba al $100 \%$ desde 20 minutos antes de la premedicación y se mantenía durante el periodo de estudio y en el periodo inmediato de recuperación. En caso de que la hubiere, la presión positiva al final de la espiración (positive end expiratory pressure [PEEP]) se mantenía a $5 \mathrm{mmHg}$ durante el procedimiento. En los pacientes con ventilación no invasiva (BiPAP vision, Respironics, Murrysville, PA, USA) se mantenía el modo presión positiva binivel (bilevel possitive airway pressure [BiPAP] $)^{11}$.

Las técnicas endoscópicas realizadas incluían: broncoaspirado difuso (BAS), lavado broncoalveolar (LBA) (150 cc de suero fisiológico distribuido en tres alícuotas de 50 cc cada una y realizado en el lóbulo donde se localizaba el infiltrado pulmonar o en el lóbulo medio o la língula cuando el infiltrado era difuso), biopsias bronquiales y biopsias transbronquiales. Las muestras obtenidas durante el estudio endoscópico fueron individualizadas en cada caso. Las técnicas para el procesamiento de las muestras microbiológicas y la interpretación de los resultados han sido descritas previamente $^{12}$.

\section{Recogida de datos y análisis}

Las indicaciones para la realización de la FBS fueron divididas en diagnósticas y terapéuticas. El impacto de la FBS en el manejo del paciente fue evaluado en función de los resultados obtenidos mediante el procedimiento. Se consideró que un estudio endoscópico era positivo cuando como resultado del procedimiento se llegaba a un diagnóstico definitivo. Se recogió la mortalidad global en la UVIR y la de los pacientes a los que se les había practicado una FBS.

Las complicaciones de la FBS se definieron como aquellos eventos ocurridos durante el procedimiento y/o $24 \mathrm{~h}$ después de finalizado. Los efectos adversos fueron recogidos prospectivamente; para ello se valoraron: la fiebre, la desaturación de oxihemoglobina con necesidad de incremento de oxígeno durante las cuatro horas posteriores al procedimiento, la presencia de sangrado que precisara finalizar el estudio, hipotensión con requerimiento o no de reposición de líquidos o uso de aminas vasoactivas, la aparición de crisis hipertensiva que obligara al inicio de tratamiento y/o detención del procedimiento y la evidencia de arritmias cardíacas durante y una hora después de la finalización de la técnica. Se registró el tiempo utilizado desde el inicio hasta el final de la exploración endoscópica y se dividieron las exploraciones según hubieran tardado menos de cinco minutos, entre cinco y diez y más de diez minutos.

Para el análisis estadístico las variables cuantitativas se expresaron como media \pm desviación estándar. Para las variables cualitativas se utilizaron porcentajes y frecuencias absolutas. Las proporciones se compararon mediante test de chi-cuadrado. Un valor de $p<0,05$ se consideró como estadísticamente significativo. Los datos fueron procesados mediante SPSS (Microsoft; Redmond, WA, EE.UU.).

\section{Resultados}

\section{Características de los pacientes}

Durante el periodo de inclusión, se admitieron 297 pacientes en la UVIR. Se realizaron 107 FBS a 69 pacientes $(23 \%$ del total de pacientes ingresados en la UVIR), 46 de los cuales eran varones. La edad media del grupo fue $60 \pm 14$ años. A 47 pacientes se les practicó una FBS; a 19 pacientes dos; a un paciente tres; a otro paciente cinco y a dos pacientes se les realizaron siete procedimientos. En las tablas 1 y 2 se muestran las indicaciones de ingreso en la UVIR y las comorbilidades de los pacientes que precisaron FBS.

\section{Indicaciones para la realización de la fibrobroncoscopia}

La broncoscopia se realizó con objetivos diagnósticos en 88 ocasiones (82\%) y terapéuticos en $19(18 \%)$. La radiografía de tórax fue normal en 25 de las 88 ocasiones (28\%) en que la FBS se llevó a cabo por motivos diagnósticos; se observaron infiltrados unilaterales en 24 casos $(27 \%)$ e infiltrados bilaterales en 39 (44\%).

Las indicaciones clínicas para la realización de una FBS diagnóstica se muestran en la tabla 3. Todas las FBS terapéuticas se llevaron a cabo como tratamiento de retención de secreciones y atelectasias; excepto en un paciente, en que

Tabla 1 Criterios de admisión en la unidad de vigilancia intensiva respiratoria de los 69 pacientes a los que se les realizó fibrobroncoscopia

\begin{tabular}{lc}
\hline Indicación & N (\%) \\
\hline Neumonía & $26(38)$ \\
Exacerbación EPOC & $17(25)$ \\
Post-cirugía torácica & $8(11)$ \\
Insuficiencia respiratoria aguda & $5(7)$ \\
Hemoptisis & $4(6)$ \\
Shock séptico & $3(4)$ \\
Otras cirugías $_{\text {Otros }^{\text {a }}}$ & $2(3)$ \\
\hline
\end{tabular}

a Otras indicaciones fueron: embolismo pulmonar, empiema, endocarditis y shock cardiogénico. 
Tabla 2 Comorbilidades de los pacientes a los que se les realizó fibrobroncoscopia

\begin{tabular}{lc}
\hline Comorbilidad & $\mathrm{N}(\%)$ \\
\hline Inmunosupresión: & $20(29)$ \\
VIH+ & $5(7)$ \\
TPH & $6(9)$ \\
Leucemia/linfoma & $6(6)$ \\
Tratamiento crónico con glucocorticoides & $2(3)$ \\
Post-quimioterapia & $1(1)$ \\
EPOC & $18(26)$ \\
Neoplasia pulmonar & $10(14)$ \\
Enfermedad cardíaca & $10(14)$ \\
\hline EPOC: enfermedad pulmonar obstructiva crónica; TPH: tras- \\
plante de progenitores hematopoyéticos; VIH: virus de la \\
inmunodeficiencia humana.
\end{tabular}

Tabla 3 Indicaciones clínicas para la realización de fibrobroncoscopia diagnóstica

\begin{tabular}{lrr}
\hline & $\mathrm{N}$ & $\%$ \\
\hline Diagnóstico de infiltrados pulmonares: & 44 & 51 \\
Pacientes inmunodeprimidos & 24 & \\
Pacientes inmunocompetentes & 20 & \\
Control post-traqueostomía & 17 & 19 \\
Control localización stents traqueobronquiales & 7 & 7 \\
Control posición tubo orotraqueal & 6 & 7 \\
Hemoptisis & 5 & 5 \\
Neoplasia endobronquial & 4 & 5 \\
Evaluación de la vía respiratoria superior & 2 & 2 \\
Neumonía aspirativa & 1 & 1 \\
Estenosis endobronquial & 1 & 1 \\
Control post-neumonectomía & 1 & 1 \\
\hline
\end{tabular}

en el contexto de una fístula broncopleural, fue necesaria la colocación de un catéter de Fogarty.

\section{Características de la fibrobroncoscopia en la unidad de vigilancia intensiva respiratoria}

Setenta y tres (68\%) de las 107 FBS se realizaron en pacientes con ventilación mecánica y 34 (32\%) fueron en pacientes con respiración espontánea y oxigenoterapia. En seis casos (6\%), el procedimiento se llevó a cabo en pacientes con ventilación no invasiva.

La FBS fue realizada por médicos internos residentes de Neumología (siempre bajo la supervisión de un neumólogo) en 72 casos (67\%), por endoscopistas en 28 casos (26\%) y por un neumólogo en siete ocasiones (6\%). En el 53\% de las FBS la duración fue menor de cinco minutos, en el $31 \%$ entre cinco y diez minutos y solo en el $16 \%$ de los casos el procedimiento duró más de diez minutos.

\section{Rentabilidad diagnóstica}

En 68 casos (64\%) se practicaron diferentes métodos broncoscópicos diagnósticos. El LBA y el BAS fueron las técnicas más comunes empleadas (ambas técnicas se realizaron en 34 ocasiones, $32 \%)$. Solo en siete casos (7\%) se obtuvieron muestras de biopsia.

En 45 de las 88 FBS diagnósticas (51\%) el objetivo era el diagnóstico del agente etiológico causante de una neumonía. Mediante la FBS se obtuvo el diagnóstico específico en 19 de los 45 casos (42\%). Específicamente, 20 FBS se realizaron en 18 pacientes inmunocompetentes (10 en pacientes con neumonía comunitaria y ocho en pacientes con neumonía asociada al ventilador) y 25 en 15 pacientes con diferentes tipos de inmunosupresión (tabla 4). En el grupo de pacientes inmunocompetentes, se obtuvo un diagnóstico etiológico específico en seis de las 20 exploraciones realizadas (30\%). Por el contrario, la rentabilidad diagnóstica de la FBS en los pacientes inmunodeprimidos fue significativamente mejor,

Tabla 4 Pacientes inmunodeprimidos con un diagnóstico específico $(n=12)$

\begin{tabular}{lllll}
\hline Edad/sexo & Causa de inmunosupresión & Técnica FBS & Diagnóstico & Éxitus \\
\hline $70 / \sigma^{\text {ra }}$ & DM/TGC & LBA & P. jirovecci \\
$70 / \sigma^{\text {ra }}$ & DM/TGC & LBA/BTB & P. jirovecci & Sí \\
$48 / q$ & MM/Auto-TPH & LBA & CMV & Sí \\
$48 / q$ & MM/Auto-TPH & LBA & VEB & Sí \\
$44 / \sigma^{7}$ & LNH/Alo-TPH & LBA & S. maltophila & Sí \\
$63 / \sigma^{7}$ & Neutropenia post-quimioterapia & LBA/BTB & Aspergillus & Sí \\
$38 / \sigma^{7}$ & LAM & LBA & P. jirovecci & No \\
$44 / q$ & LAM/Alo-TPH & LBA & S. maltophila+ Aspergillus \\
$66 / \sigma^{7}$ & LNH/Auto-TPH & LBA & Aspergillus & SI \\
$50 / q$ & VIH+ & LBA & SARM & SI \\
$54 / \sigma^{7}$ & VIH+ & LBA & P. aeruginosa & SI \\
$64 / q$ & LM/Auto-TPH & LBA & VHS & NO \\
\hline
\end{tabular}

BTB: biopsia transbronquial; CMV: citomegalovirus; DM: dermatomiositis; LAM: leucemia aguda mieloide; LBA: lavado broncoalveolar; LNH: linfoma no Hodgkin; MM: mieloma múltiple; SARM: S. aureus resistente a meticilina; TGC: tratamiento glucocorticoideo crónico; TPH: trasplante de progenitores hematopoyéticos; VEB: virus de Epstein-Barr; VHS: virus herpes simple; VIH: virus de la inmunodeficiencia humana.

a Dos exploraciones en el mismo paciente. 
Tabla 5 Pacientes inmunocompetentes con un diagnóstico específico $(n=6)$

\begin{tabular}{lllll}
\hline Edad/sexo & Causa de inmunosupresión & Técnica FBS & Diagnóstico & Éxitus \\
\hline $66 / \sigma^{7}$ & EPOC & BAS & MAC & No \\
$79 / \sigma^{7}$ & EPOC & BAS/BB & P. aeruginosa/Herpes simple & No \\
$51 / \sigma^{7}$ & EPOC & BAS & SARM & No \\
$65 / 9$ & Neoplasia pulmonar & LBA/BTB & Carcinomatosis & No \\
$51 / \sigma^{7}$ & Insuficiencia cardíaca & LBA/BTB & Candidiasis & Sí \\
$52 / \sigma^{7}$ & EPOC & LBA & SARM & No \\
\hline
\end{tabular}

BAS: broncoaspirado; BB: biopsia bronquial; BTB: biopsia transbronquial; EPOC: enfermedad pulmonar obstructiva crónica; LBA: lavado broncoalveolar; MAV: M. avium complex; SARM: S. aureus resistente a meticilina.

logrando un diagnóstico específico en 12 de las 25 exploraciones realizadas $(48 \%)(p<0,01)$, (tablas 4 y 5$)$.

\section{Seguridad y evolución}

La FBS no precipitó la necesidad de ventilación mecánica en ningún paciente con respiración espontánea o ventilación no invasiva. No hubo diferencias en la $\mathrm{PaO}_{2} / \mathrm{FiO}_{2}$ antes y después del procedimiento (tabla 6). Los cambios en la $\mathrm{PaO}_{2} / \mathrm{FiO}_{2}$ no fueron significativos cuando se dividieron los pacientes entre aquellos con ventilación mecánica y los que tenían respiración espontánea, o aquellos con infiltrados pulmonares unilaterales o bilaterales. En cambio, en aquellos pacientes en los que se realizó un LBA se observó un descenso significativo de la $\mathrm{PaO}_{2} / \mathrm{FiO}_{2}$ después del procedimiento ( $182 \pm 74$ vs $163 \pm 79 ; p<0,005)$, tanto en el grupo de pacientes inmunodeprimidos $(n=20)(203 \pm 79$ vs $182 \pm 86$; $p<0,02)$ como en los inmunocompetentes $(n=14)(140 \pm 39$ vs $125 \pm 42 ; \mathrm{p}<0,02)$.

En $13(12 \%)$ de las 107 FBS fue necesario un incremento transitorio de la $\mathrm{FiO}_{2}$ (con respecto a la $\mathrm{FiO}_{2}$ que recibía el paciente antes de la exploración endoscópica), como resultado del empeoramiento en la oxihemoglobina durante el procedimiento. En 10 casos (9\%) se detectaron eventos cardiorrespiratorios reversibles (extrasístoles supraventriculares aisladas y/o variaciones en la presión arterial), sin requerimiento de fármacos. No se identificaron otras complicaciones significativas en las horas próximas al procedimiento.

La mortalidad global de los pacientes admitidos en la UVIR durante el periodo del estudio fue del 14\% (42 de los 297 pacientes admitidos). En el grupo de pacientes que no requirieron $\mathrm{FBS}$ la mortalidad fue del $11 \%$, detectándose una mortalidad del $25 \%$ en el grupo de pacientes a los que se les realizó al menos una FBS (17 de los 68 pacientes incluidos). La mortalidad fue del 15\% (7 de 46 pacientes) en aquellos pacientes que precisaron una FBS y del $45 \%$ en los pacientes que requirieron más de una FBS (10 de 22 pacientes).

La mortalidad en los pacientes inmunodeprimidos a los que se les realizó una FBS diagnóstica fue del 60\% (nueve de 15 pacientes), significativamente superior a la mortalidad observada en el grupo de pacientes inmunocompetentes donde fue del 30\% (6 de 20 pacientes) $(p<0,05)$. En siete de nueve pacientes inmunodeprimidos que fallecieron, el diagnóstico específico se obtuvo mediante FBS.

\section{Discusión}

El presente estudio demuestra que la FBS es un procedimiento que se indica con frecuencia en la UVIR y que puede llevarse a cabo de forma rápida en pacientes críticos, sin complicaciones mayores y obteniéndose una buena rentabilidad diagnóstica. La necesidad de FBS repetidas durante el ingreso en la UVIR es un signo indirecto de mal pronóstico.

Se trata del primer estudio prospectivo observacional realizado en una unidad especializada de cuidados intensivos respiratorios, con una alta prevalencia de enfermedad pulmonar aguda y crónica. La originalidad del presente trabajo reside en conocer las indicaciones de la FBS y la rentabilidad y potenciales efectos deletéreos en estas circunstancias. Así, Dunagan et al. mostraron que solo al $0,5 \%$ de los pacientes admitidos en una unidad coronaria se les realizaba una $\mathrm{FBS}^{9}$. En cambio, en el presente estudio, el $23 \%$ de los pacientes requirieron una FBS en algún momento del ingreso. La indicación más frecuente de FBS en la UVIR fue el estudio de infiltrados pulmonares que, de forma interesante, en su mayoría afectaban a pacientes con alguna inmunosupresión de base (tabla 3). El diagnóstico mediante FBS en

Tabla 6 Gasometría arterial pre-FBS y post-FBS en la población evaluada $(n=69)$

\begin{tabular}{|c|c|c|c|}
\hline & $\mathrm{PaO}_{2} / \mathrm{FiO}_{2}$ pre-FBS & $\mathrm{PaO}_{2} / \mathrm{FiO}_{2}$ post-FBS & $\mathrm{p}$ \\
\hline Todos los pacientes & $209 \pm 98$ & $206 \pm 103$ & NS \\
\hline Pacientes con VM & $208 \pm 100$ & $208 \pm 102$ & NS \\
\hline Pacientes con ventilación espontánea ${ }^{a}$ & $211 \pm 88$ & $203 \pm 105$ & NS \\
\hline Pacientes con insuficiencia respiratoria severa ${ }^{\dagger}$ & $157 \pm 45$ & $158 \pm 43$ & NS \\
\hline Pacientes que precisaron LBA & $182 \pm 74$ & $163 \pm 79$ & 0,005 \\
\hline
\end{tabular}


el grupo de pacientes inmunodeprimidos fue mayor que el obtenido en los pacientes inmunocompetentes (48\% vs $30 \%$; $\mathrm{p}<0,01)$. A pesar de ello, la mortalidad en el primer grupo fue mayor, tal como se ha observado en otras series ${ }^{13-15}$. Aunque el ingreso en $\mathrm{UCl}$ de pacientes oncológicos con diferentes tipos de inmunosupresión se cuestionó durante años por su elevada tasa de mortalidad, estudios recientes han demostrado una mejoría significativa de la supervivencia. De este modo, Lecuyer et al. en un estudio reciente en que se evaluaron 188 pacientes con cáncer, que precisaron ventilación mecánica, demostraron un $20 \%$ de supervivencia ${ }^{16}$. En nuestro estudio, seis de 15 pacientes inmunodeprimidos a los que se les realizó una FBS sobrevivieron después de su estancia en la UVIR (40\% de supervivencia).

La seguridad es uno de los aspectos más relevantes a la hora de decidir la realización de una FBS en una UCI. Diferentes estudios han mostrado que la FBS puede tener efectos adversos sobre el enfermo crítico, incluyendo alteraciones hemodinámicas, gasométricas o en la mecánica respiratoria ${ }^{5-7}$. Aunque dichos efectos pueden ser significativos, probablemente no sean clínicamente relevantes en la mayoría de los casos. Así, Olopade et al. tras valorar 1.150 FBS realizadas en la $\mathrm{UCl}$, detectaron tan solo siete complicaciones transitorias ${ }^{17}$. En nuestro estudio y considerando la presencia común de enfermedad pulmonar crónica, la hipoxemia significativa no fue evidente ni siquiera en el subgrupo de pacientes con mayor afectación respiratoria $\left(\mathrm{PaO}_{2} / \mathrm{FiO}_{2}\right.$ ratio <250) (tabla 6). Igualmente, se observó una baja frecuencia de eventos cardiovasculares, como han mostrado estudios previos ${ }^{9,18}$. El escaso tiempo empleado en la realización del procedimiento, quizás justifique en parte la ausencia de efectos adversos significativos en nuestros pacientes ${ }^{19}$. Una FBS realizada por residentes en formación y supervisada por un senior o endoscopista experimentado puede llevarse a cabo de forma rápida y sencilla, particularmente cuando el procedimiento tiene fines diagnósticos. En nuestra serie el $53 \%$ de las exploraciones se realizaron en menos de cinco minutos.

La ausencia de efectos adversos significativos mencionados previamente contrasta con la experiencia de otros autores. Trouillet et al., en una serie de 107 pacientes ventilados, evidenciaron un descenso de la $\mathrm{PaO}_{2}$ tras la FBS en un $26 \%$ de los $\operatorname{casos}^{20}$. Si bien, y tal como los autores reconocen, el empleo de agentes bloqueantes neuromusculares además de la sedación podría haber evitado asincronías del paciente con el ventilador y los consecuentes descensos significativos de la $\mathrm{PaO}_{2}{ }^{21}$.

En nuestro estudio los pacientes a los que se les practicó un LBA experimentaron un cambio significativo en la $\mathrm{PaO}_{2} / \mathrm{FiO}_{2}$. Diferentes autores, incluido nuestro propio grupo, han demostrado que la realización de un LBA en un paciente ventilado puede causar cambios en el intercambio de gases incluso $24 \mathrm{~h}$ después de finalizado el procedimiento $8,22,23$. Aunque este hecho se ha de tener en cuenta, hay que recordar que el LBA es la mejor técnica diagnóstica para el estudio de los infiltrados pulmonares, especialmente en los pacientes inmunodeprimidos. Muy recientemente Azoulay et al. han publicado el primer estudio controlado aleatorizado en pacientes inmunodeprimidos con insuficiencia respiratoria aguda, mostrando que la FBS es un procedimiento rentable cuando se hace de forma precoz tras la admisión en la $\mathrm{UCl}$, sin incrementar la necesidad de intubación ${ }^{24}$. En este sentido, la mayor mortalidad evidenciada en los pacientes a los que se les realizan diversas broncoscopias, no debe adscribirse a la exploración en sí misma, sino que muy probablemente su requerimiento repetido traduce una mayor gravedad de la complicación pulmonar.

Una limitación potencial de nuestro estudio es la ausencia de criterios objetivos para la indicación de la FBS. Debido a que la FBS se indicaba según el criterio del médico responsable, no puede excluirse que la selección de pacientes podría haber influido parcialmente en los resultados. En este sentido, la realización de la FBS en los enfermos más graves podría incrementar los efectos adversos. No obstante, la indicación de FBS era realizada por neumólogos con experiencia en dicha técnica y que seguían las guías establecidas para el diagnóstico de la insuficiencia respiratoria ${ }^{12}$.

En conclusión, la FBS es una técnica que se utiliza con frecuencia en la UVIR, en la mayoría de los casos con fines diagnósticos. Es un procedimiento rápido y seguro que contribuye sustancialmente al manejo del paciente crítico. Los pacientes que durante su estancia en la UVIR requieren FBS adicionales tienen una mortalidad más elevada, probablemente como marcador indirecto de una complicación pulmonar grave.

\section{Conflicto de intereses}

Los autores declaran que no existen conflictos de intereses.

\section{Bibliografía}

1. Pue CA, Pacht ER. Complications of fiberoptic bronchoscopy at a university hospital. Chest. 1995;107:430-2.

2. Turner JS, Willcox PA, Hayhurst MD, Potgieter PD. Fiberoptic bronchoscopy in the intensive care unit: a prospective study of 147 procedures in 107 patients. Crit Care Med. 1994;22:259-64.

3. Thiery G, Azoulay E, Darmon M, Ciroldi M, De Miranda S, Lévy V, et al. Outcome of cancer patients considered for intensive care unit admission: A hospital-wide prospective study. J Clin Oncol. 2005;23:4406-13.

4. Esteban A, Anzueto A, Frutos F, Alía I, Brochard L, Stewart TE, et al. Characteristics and outcomes in adult patients receiving mechanical ventilation. A 28-day international study. JAMA. 2002;287:345-55.

5. Hsia D, DiBlasi RM, Richardson P, Crotwell D, Debley J, Carter E. The effects of flexible bronchoscopy on mechanical ventilation in a pediatric lung model. Chest. 2009;135:33-40.

6. Bush A. Primum Non Nocere. How to cause chaos with a bronchoscope in the ICU. Chest. 2009;135:2-3.

7. Davis L, Mister R, Spence DPS, Calverley PMA, Earis JE, Pearson MG. Cardiovascular consequences of fiberoptic bronchoscopy. Eur Respir J. 1997;10:695-8.

8. Bauer TT, Torres A, Ewig S, Hernández C, Sanchez-Nieto JM, Xaubet $A$, et al. Effects of bronchoalveolar lavage volume on arterial oxygenation in mechanically ventilated patients with pneumonia. Intensive Care Med. 2001;27:384-93.

9. Dunagan DP, Burke HL, Aquino SL, Chin R, Adair NE, Haponik EF. Fiberoptic bronchoscopy in coronary care unit patients. Chest. 1998;114:1660-7.

10. Babb J, Bowie P, Brewin A, Fraise A, Garrard C, Harvey J, et al. British Thoracic Society guidelines on diagnostic flexible bronchoscopy. Thorax. 2001;56 Suppl 1:i1-21.

11. Antonelli M, Conti G, Rocco M, Arcangeli A, Cavaliere F, Proietti $\mathrm{R}$, et al. Noninvasive positive-pressure ventilation vs. 
Conventional oxygen supplementation in hypoxemic patients undergoing diagnostic bronchoscopy. Chest. 2002;121:1149-54.

12. Rañó A, Agustí C, Jimenez P, Angrill J, De Benito N, Danés C, et al. Pulmonary infiltrates in non-HIV IC patients. A diagnostic approach using non-invasive and bronchoscopic techniques. Thorax. 2001;56:379-87.

13. Nichols DJ, Maziarz RT, Haupt MT. Mechanical ventilation in hematopoietic stem cell transplant recipients: is there need for reevaluation? Chest. 1999;116:857-9.

14. Azolay E, Thiery G, Chevret S, Moreau D, Darmon M, Bergeron A, et al. The prognosis of acute respiratory failure in critically ill cancer patients. Medicine. 2004;83:360-70.

15. Raoof ND, Groeger JS. You never know: One of your patients with cancer might surprise you. Crit Care Med. 2007;35:965-6.

16. Lecuyer L, Chevret S, Thiery G, Darmon M, Schlemmer B, Azoulay E. The ICU Trial: A new admission policy for cancer patients requiring mechanical ventilation. Crit Care Med. 2007;35:808-14.

17. Olopade CO, Prakash UB. Bronchoscopy in the critical-care unit. Mayo Clin Proc. 1989;64:1255-63.

18. Dweik RA, Mehta AC, Meeker DP, Arroliga A. Analysis of the safety of bronchoscopy after recent acute myocardial infarction. Chest. 1996;110:825-8.
19. Wood RE. Pitfalls in the use of the flexible bronchoscope in pediatric patients. Chest. 1990;97:199-203.

20. Trouillet JL, Guiguet M, Gibert C, Fagon JY, Dreyfuss D, Blanchet $\mathrm{F}$, et al. Fiberoptic bronchoscopy in ventilated patients. Evaluation of cardiopulmonary risk under midazolam sedation. Chest. 1990;97:927-33.

21. Papazian L, Forel JM, Gacouin A, Penot-Ragon C, Perrin G, Loundou A, et al. Neuromuscular blockers in early acute respiratory distress syndrome. N Engl J Med. 2010;363: 1107-16.

22. Steinberg KP, Mitchell DR, Maunder RJ, Milberg JA, Whitcomb ME, Hudson LD. Safety of bronchoalveolar lavage in patients with adult respiratory distress syndrome. Am Rev Respir Dis. 1993;148:556-61.

23. Kollef MH, Bock KR, Richards RD, Hearns ML. The safety and diagnosis accuracy of minibronchoalveolar lavage in patients with suspected ventilator associated pneumonia. Ann Intern Med. 1995;122:743-8.

24. Azoulay E, Mokart D, Lambert J, Lemiale V, Rabbat A, Kouatchet $A$, et al. Diagnostic strategy for hematology and oncology patients with acute respiratory failure. Randomized controlled trial. Am J Resp Crit Care Med. 2010;182: 1038-46. 